\title{
Endothelial Dysfunction Occurs prior to Clinical Evidence of Polycystic Kidney Disease
}

\author{
Karen M. Peterson ${ }^{a}$ Federico Franchi ${ }^{a}$ Darrel L. Loeffler ${ }^{a}$ Peter J. Psaltis ${ }^{a}$ \\ Peter C. Harris ${ }^{b}$ Lilach O. Lerman ${ }^{b} \quad$ Amir Lerman ${ }^{a}$ Martin Rodriguez-Porcel ${ }^{a}$ \\ Divisions of a Cardiovascular Diseases and ${ }^{b}$ Nephrology and Hypertension, Department of Internal Medicine, \\ Mayo Clinic, Rochester, Minn., USA
}

\section{Key Words}

Acetylcholine - Autosomal inheritance - Cardiovascular disease $\cdot$ Endothelial dysfunction · Polycystic kidney disease

\begin{abstract}
Objective: Polycystic kidney disease (PKD), a monogenic disease with an autosomal dominant or an autosomal recessive form of inheritance (ARPKD), is the most common genetic cause of renal dysfunction and end-stage renal failure. In addition to the development of cysts, the autosomal form of PKD is associated with vascular endothelial dysfunction, a marker of vascular disease. Whether vascular endothelial dysfunction is also present in ARPKD, and its relationship with renal dysfunction remain to be determined. Methods: ARPKD rats (PCK model) and controls were studied at 6 and 10 weeks of age, and mean arterial pressure and renal function were measured. Aortic endothelial function was assessed using organ chamber techniques. Aortic endothelial cells (ECs) were isolated, characterized and their function studied. Results: Compared to controls, ARPKD animals had a decrease in the vasorelaxation to endothelium-dependent vasodilators, even prior to changes in mean arterial pressure or renal function. The abnormal vasoreactivity was corrected
\end{abstract}

with L-arginine (a precursor of nitric oxide, NO), while the expression of endothelial NO synthase (eNOS) was unchanged. Furthermore, isolated ECs from 6-week-old ARPKD animals showed increased oxidative stress, with preserved eNOS expression and abnormal patterns of migration and angiogenic capacity (measured by the scratch and tube formation assays, respectively). Conclusion: ARPKD leads to impairments in aortic vascular function and ECs at an early stage, which can have significant functional consequences, potentially representing a novel therapeutic target in this disease.

(c) 2013 S. Karger AG, Basel

\section{Introduction}

Polycystic kidney disease (PKD) is the most common renal monogenic disorder and an important cause of endstage renal disease $[1,2]$. PKD is characterized by abnormal production of cytoskeletal proteins that are important for the normal function of primary cilia, i.e. cellular structures that determine the orientation, shape and function of somatic cells $[1,2]$. PKD is characterized by a defect in the cilium-associated proteins polycystin-1 and

\section{KARGER}

E-Mail karger@karger.com www.karger.com/ajn
(C) 2013 S. Karger AG, Basel

0250-8095/13/0383-0233\$38.00/0
Martin Rodriguez-Porcel, MD

200 First Street SW

Rochester, MN 55905 (USA)

E-Mail rodriguez.m@mayo.edu 
-2 in its autosomal dominant form, while the autosomal recessive form of the disease has been linked to a defect in the Pkhd1 gene and in the expression of the ciliumassociated protein fibrocystin $[2,3]$. PKD is a chronic disease mainly characterized by the development of parenchymal cysts of tubular origin, which leads to impairments in the handling of the urine concentration ability and end-stage renal function $[1,4]$. Despite differences in transmission and genes involved, both forms have been associated with cyst development and renal dysfunction, albeit with some differences in the rate of progression of the disease $[2,3]$.

In addition to the renal tubular defects, PKD has also been associated with abnormalities in the vasculature $[5$, 6]. Previous studies have shown that PKD patients and different animal models of PKD have abnormal response to hyperemia and endothelial-dependent vasorelaxation $[5,6]$, which can contribute to the hypertension observed in PKD patients. Furthermore, this defect has been linked to a deficiency in nitric oxide (NO) bioavailability by endothelial cells (ECs) [6]. Because abnormalities in vascular function have been identified as precursors of more advanced vascular disease $[7,8]$, identification of the mechanisms that may lead to vascular dysfunction and early treatment can be of significant importance. Until now, these abnormalities have been demonstrated mainly in the autosomal dominant form of PKD (ADPKD). Whether the autosomal recessive form of PKD (ARPKD) is also associated with vascular abnormalities, i.e. endothelial dysfunction, and its relation to the appearance of renal dysfunction remain to be determined. Understanding the pathophysiology and timing of vascular changes in $\mathrm{PKD}$ may provide novel therapeutic targets for the treatment of this disease.

Thus, in this study, we tested the hypothesis that the PCK rat model of ARPKD has vascular endothelial dysfunction, its relationship with renal dysfunction and the role of ECs in disease pathophysiology.

\section{Materials and Methods}

\section{Study Outline}

All procedures using animals were reviewed and approved by the Mayo Foundation Institutional Animal Care and Use Committee. Female PKD (PCK rat model, $\mathrm{n}=9$, which is on a SpragueDawley background) [9] and wild-type (WT) controls (SpragueDawley, $\mathrm{n}=10$ ) were classified into early (5-6 weeks of age) and late (10-12 weeks of age). Systolic blood pressure was measured noninvasively using the tail cuff method [10], while renal blood flow was measured noninvasively using high-resolution ultra- sound (Vevo770; Visualsonics, Toronto, Ont., USA) [11]. Aortic rings were obtained for studies of vascular reactivity using organ chamber techniques [12]. Another set of animals was used to isolate aortic ECs for cell culture experiments. The isolation of ECs was performed using the previously described explant technique, and the phenotype of ECs was performed using standard RT-PCR and Western blotting techniques. Cell viability and cell proliferation were done using lactate dehydrogenase $(\mathrm{LDH})$ release and 3-(4,5-dimethylthiazol-2-yl)-2,5-diphenyltetrazolium bromide) MTT assays, respectively. The angiogenic capacity and migration of ECs were assessed by the tube formation assay and the 'scratch' assay, respectively. Details on the methods can be found in the online supplementary information (for all online suppl. material, see www.karger.com/doi/10.1159/000354236).

\section{Statistical Analysis}

Data are expressed as means \pm SEM or as percent change from the maximal contraction (in vitro vascular reactivity). Within each group, repeated measurements were analyzed with repeated-measure ANOVA followed by the Bonferroni t test or by unpaired Student's t test between groups. Statistical significance was accepted for a value of $\mathrm{p}<0.05$.

\section{Results}

\section{Systemic Hemodynamics and Renal Function}

At 5-6 weeks of age, there was no difference in systolic blood pressure (control: $109.90 \pm 4.93$ vs. PKD: $112.76 \pm 4.74 \mathrm{~mm} \mathrm{Hg}, \mathrm{p}=0.35$ ) or protein/creatinine ratio (control: $0.81 \pm 0.28$ vs. PKD: $0.70 \pm 0.08, \mathrm{p}=0.45$ ). However, at 10-12 weeks of age, PCK animals had a small but significant increase in systolic blood pressure compared to controls $(126.22 \pm 2.73$ vs. $116.45 \pm 3.53$ $\mathrm{mm} \mathrm{Hg}$, respectively, $\mathrm{p}=0.02$ ) together with an increase in the protein/creatinine ratio $(0.93 \pm 0.2$ vs. $0.48 \pm 0.06$, respectively, $\mathrm{p}=0.02)$. The renal perfusion of 5 - to 6 -week-old animals was not different between control and PCK animals $(3.20 \pm 0.24$ and $2.76 \pm 0.20 \mathrm{ml} / \mathrm{min} / 100$ $\mathrm{ml}$, respectively, $\mathrm{p}=0.3)$. We have previously shown that PCK animals at 12 weeks have lower global renal perfusion than controls [11].

\section{Vascular Reactivity: Role of NO}

The response of the aortic rings to cumulative concentrations of phenylephrine was not statistically different between the groups studied (online suppl. fig. S1), and there was no difference in the precontraction obtained with phenylephrine between control and PCK (nonsignificant, NS).

The vasorelaxation response to the endothelium-dependent vasodilator acetylcholine in aortic rings of $\mathrm{PKD}$ animals was significantly attenuated compared to normal 


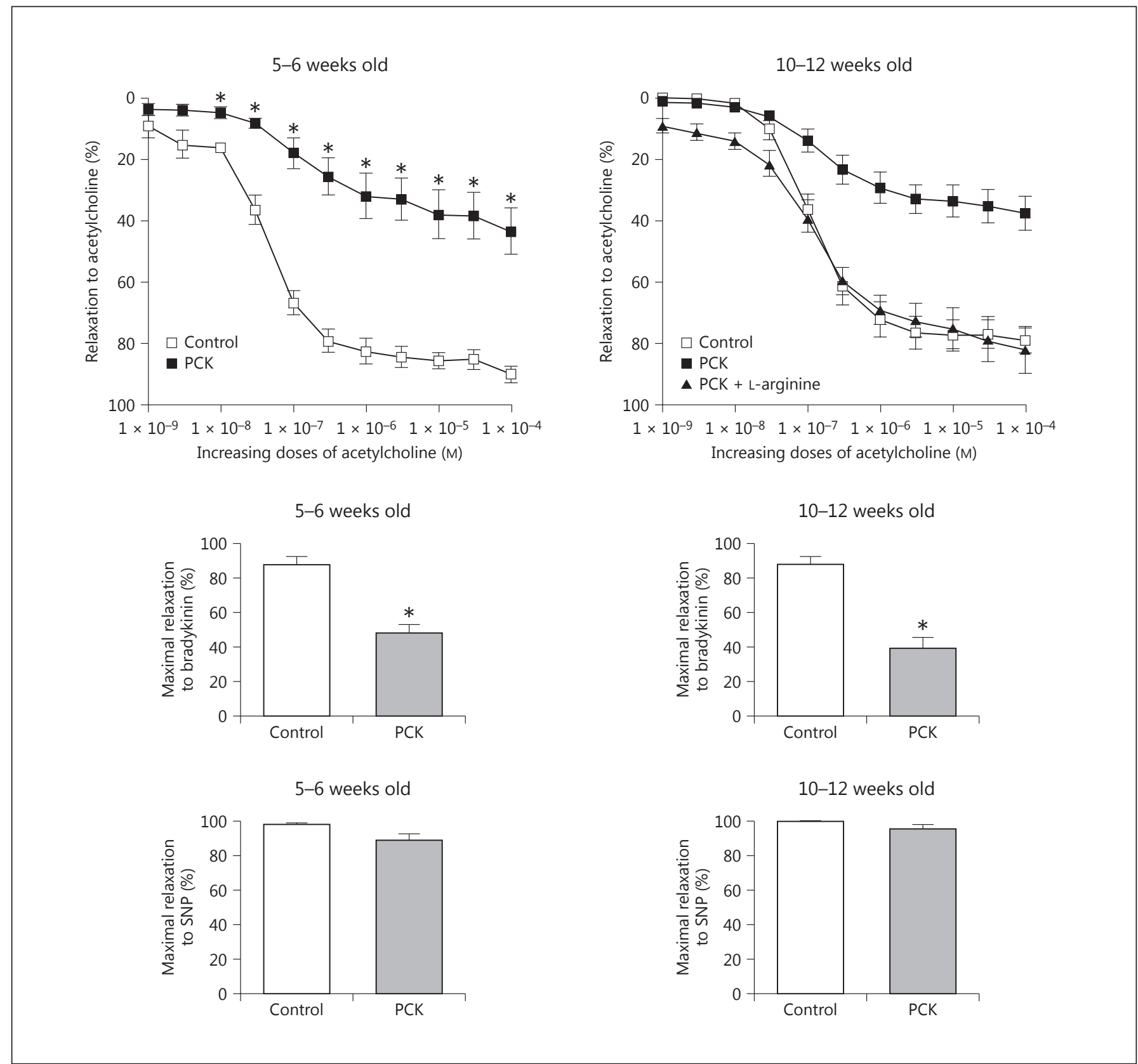

Fig. 1. Aortic vasorelaxation response to increasing doses of acetylcholine in control and PCK animals 5-6 (top left) and 10-12 weeks old (top right). Maximal vasorelaxation to bradykinin in animals 5-6 (middle left) and 10-12 weeks old (middle right).
Maximal vasorelaxation to sodium nitroprusside (SNP) in animals 5-6 (bottom left) and 10-12 weeks old (bottom right). ${ }^{*} \mathrm{p}<0.05$ vs. control and PCK $+\mathrm{L}$-arginine. animals (fig. 1, top). Importantly, the abnormal vasoreactivity response was observed not only in 10- to 12-weekold animals (fig. 1, top right), which already have a small increase in mean arterial pressure and renal dysfunction, but also in young PCK animals (fig. 1, top left), even pri- or to changes in creatinine clearance and the protein/creatinine ratio. In PCK animals of both ages, an abnormal response was also observed in response to increasing doses of bradykinin (fig. 1, middle panels). There was no difference in the vasorelaxation to the non-endothelium-de- 


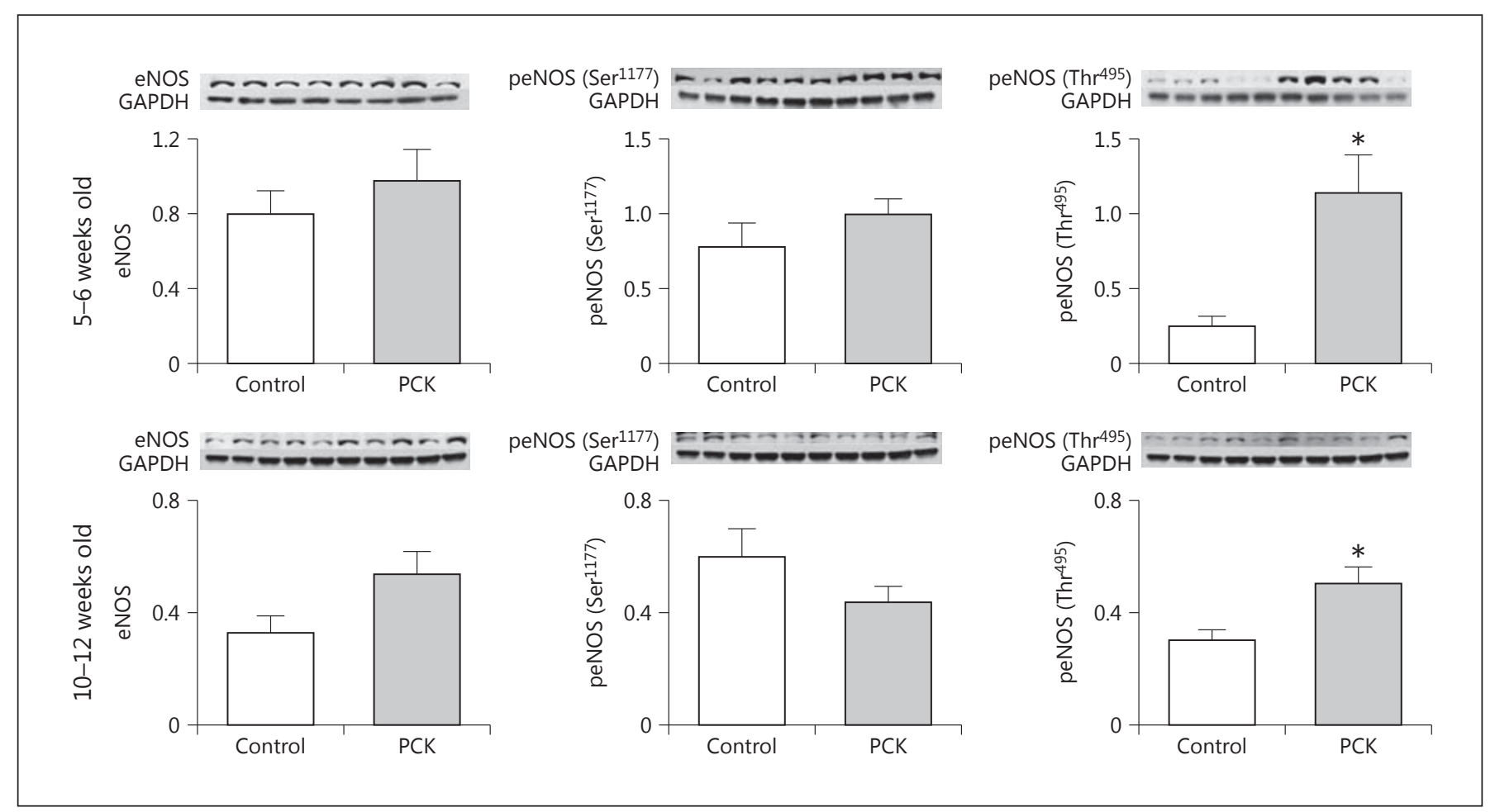

Fig. 2. Protein expression of eNOS and its active (eNOS phosphorylated at Ser $^{1177}$ ) and inactive (eNOS phosphorylated at Thr ${ }^{495}$ ) forms in 5- and 10-week-old isolated ECs showing an increase in the expression of inactive eNOS in PCK compared to control. * $\mathrm{p}<0.05$ vs. control.

Fig. 3. Protein expression of pro-oxidant enzymes in 5-week-old isolated ECs showing an imbalance towards a pro-oxidant status in PCK vs. control. ${ }^{*} \mathrm{p}<0.05$ vs. WT.

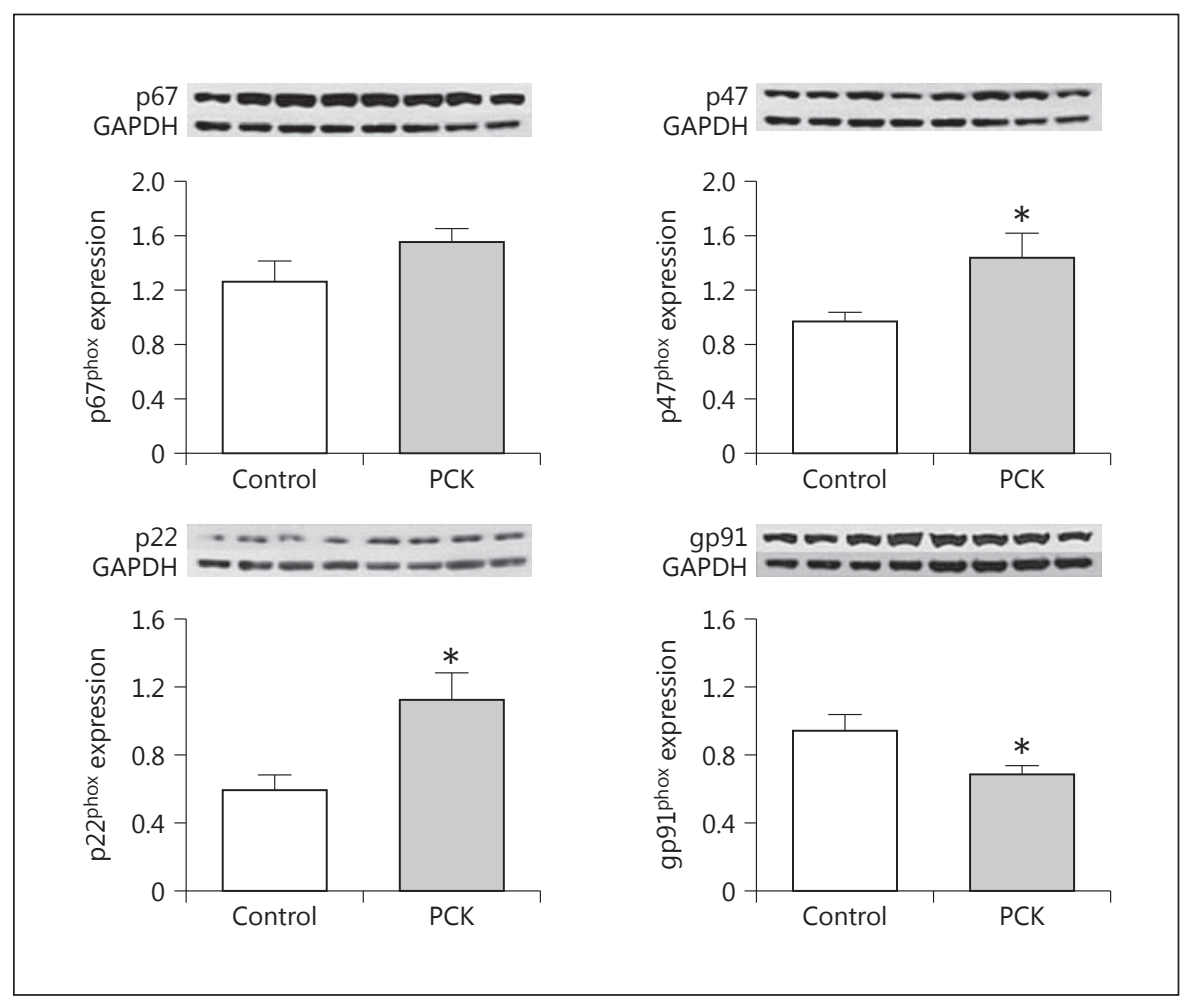

Peterson/Franchi/Loeffler/Psaltis/Harris/ Lerman/Lerman/Rodriguez-Porcel 
pendent vasodilator sodium nitroprusside between the groups at either age (fig. 1, bottom panels).

There was no difference between groups in the aortic protein expression of endothelial NO synthase (eNOS; control: $2.10 \pm 0.87$ vs. PCK: $2.95 \pm 0.90$, NS) or inducible NOS (control: $2.04 \pm 0.75$ vs. PCK: $1.80 \pm 0.65$, NS). However, when aortic rings were pre-incubated with the NO precursor L-arginine, the vasorelaxation to acetylcholine was normalized (fig. 1, top right), suggesting that a decrease in NO bioavailability was implicated in the PKD abnormal response.

\section{Isolation and Characterization of ECs}

To specifically test the function of ECs, ECs were successfully isolated from the aorta of control and PKD animals using the explant technique. Furthermore, both control and PKD ECs expressed the endothelial marker von Willebrand factor and were positive for the uptake of acetylated low-density lipoprotein (online suppl. fig. S2A), showing that the isolated cells are in fact ECs. Furthermore, ECs from both WT and PCK have the capacity to express fibrocystin (online suppl. fig. S2B), although PCK rats have a splicing mutation in the Pkhd1 gene, as previously described [13], leading to an abnormal protein and potentially abnormal cell function.

\section{NO System/Oxidative Stress Balance in Isolated Aortic} ECs

ECs of animals aged 5 and 10 weeks did not show a difference in eNOS expression between PCK and controls. Although there was no difference in the expression of the 'active' form of eNOS (phosphorylated at Ser ${ }^{1177}$ ) between the groups, the expression of the 'inactive' form of eNOS (phosphorylated at $\mathrm{Thr}^{495}$ ) was significantly higher in PCK than control rats of both age groups (5 and 10 weeks; fig. 2).

An imbalance in the expression of pro-oxidant enzymes was observed in 5-week-old PCK-ECs compared to controls but not in ECs isolated from older animals. Specifically, 5-week-old PCK-ECs had an increase in the expression of $\mathrm{NAD}(\mathrm{P}) \mathrm{H}$ p22 $2^{\text {phox }}, \mathrm{NAD}(\mathrm{P}) \mathrm{H}$ p $47^{\text {phox }}$, with no change in the expression of $\mathrm{NAD}(\mathrm{P}) \mathrm{H}$ p67 $7^{\text {phox }}$ and a decrease in $\mathrm{NAD}(\mathrm{P}) \mathrm{H}$ gp91 $91^{\text {phox }}$ (fig. 3).

\section{Functional EC Studies}

Proliferation/Death. Compared to controls, PCK-ECs had increased cell proliferation (MTT assay and expression of proliferating cell nuclear antigen, fig. 4a) and cell death (LDH release assay), although the expression of caspase 3 was not different between PKD and controls (fig. 4a).

Endothelial Function in PKD
Angiogenic Capacity. ECs from PKD animals had abnormal function, as demonstrated by an impaired angiogenic capacity, compared to control cells. This abnormal capacity is evidenced by the lower tube length formation of PCK-ECs in the first $3 \mathrm{~h}$ after plating (fig. 4b).

Migration. In response to injury, ECs from PCK animals had significantly decreased migration compared to $\mathrm{WT}$, as shown by PCK-ECs covering a smaller area after injury (fig. 4c).

\section{Discussion}

This study shows that the aorta of PCK animals, a model of PKD, has abnormal endothelial-dependent vascular reactivity probably due to a primary defect in ECs.

The pathogenesis of PKD has been mainly associated with a primary renal tubular dysfunction, although variables of vascular disease have been described (e.g. vascular aneurysms) $[4,14]$. In this study, we show that the PCK rat model of PKD, a model that is autosomal recessive by inheritance pattern, also possesses an abnormal pattern of endothelial-dependent vascular reactivity. Furthermore, we show that it is corrected by the addition of the NO precursor L-arginine, despite no changes in NOS expression, implying that the problem relates to substrate rather than NOS availability. Furthermore, PKD has been shown to have elevated levels of asymmetric dimethylarginine previously [15], which competes with L-arginine for eNOS and the production of NO. This hypothesis is supported by our finding of an increased expression of 'inactive' peNOS in ECs isolated from both 5- and 10 -week-old animals. Although we have expected a decrease in the active form, it is important to keep in mind that this balance could be tenuous and that the in vivo activation of eNOS or inducible NOS could somewhat vary from the in vitro setting. Moreover, it should be noted that the expression of NOS is dynamic, so we cannot exclude the possibility that at other time points the expression pattern of eNOS may be different. Importantly, we show that the aortic vascular dysfunction is not primarily related to renal dysfunction, preceding changes in global renal perfusion or urinary creatinine/protein ratio. The importance of our findings is that we show for the first time that the PCK rat model also presents abnormalities in non-renal vascular function and provides insights in the timing of the appearance of these abnormalities.

In this study, the abnormalities in vascular reactivity were mainly related to the endothelial-dependent vasore- 
Fig. 4. a Cell viability (LDH release assay and protein expression of caspase 3 ) and proliferation (MTT assay and protein expression of proliferating cell nuclear antigen, PCNA) in PCK and control ECs showing that PCK-ECs have increased cell death and cell proliferation compared to control. b Tube formation assay in WT and PCKECs. Left panel shows a representative picture of control and PCK-EC tube formation and the right panel shows the quantification of tube length. Note that PCK-ECs have decreased capacity to form tubes compared to control. c Migration capacity of ECs in response to injury. ECs from PCK animals have decreased capacity for migration compared to control as evidenced by representative images (left panel) and quantification of area covered by ECs (right panel). ${ }^{*} \mathrm{p}<0.05$ vs. WT.

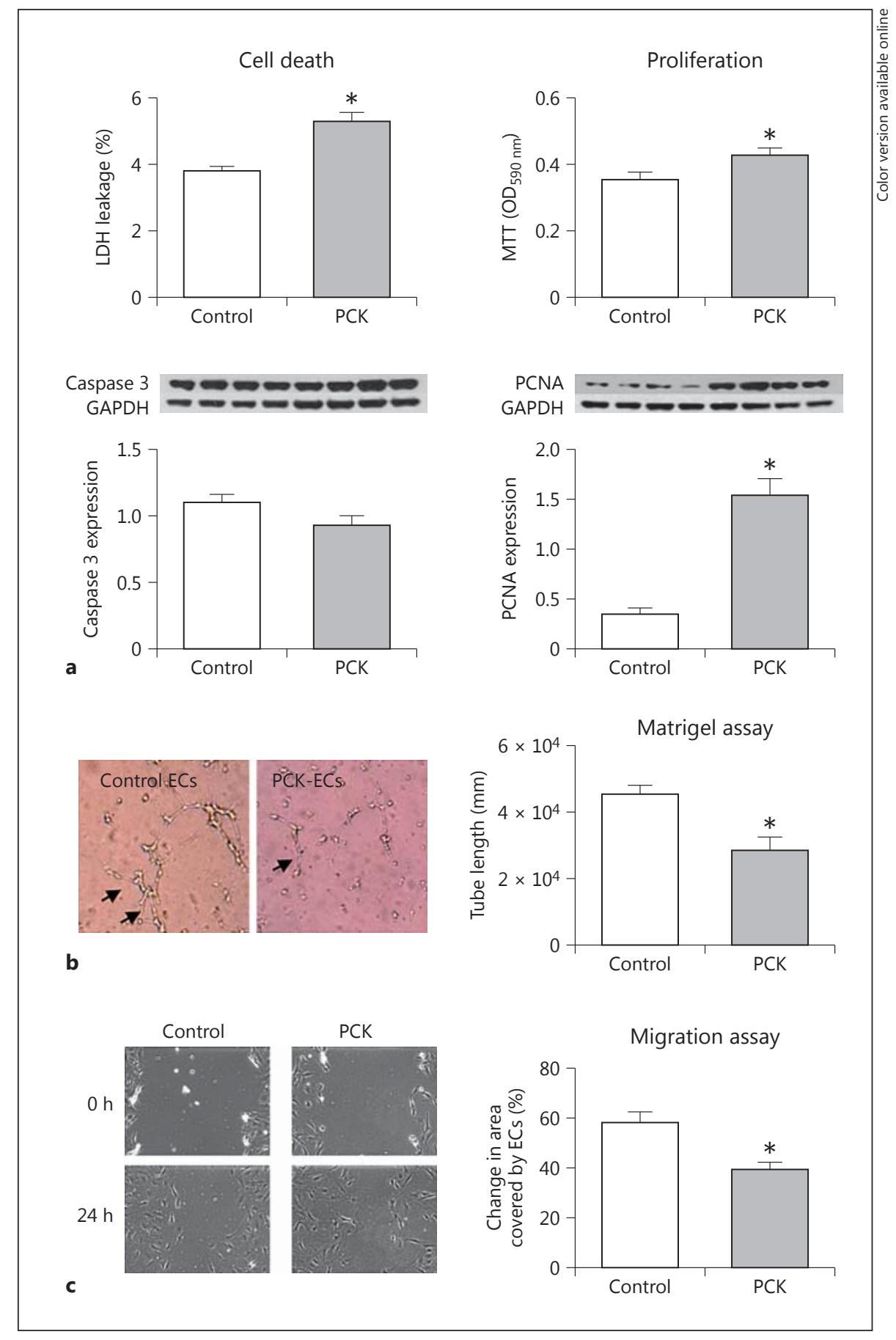

laxation, while the endothelium-independent, i.e. smooth muscle, relaxation was not impaired. This finding of early abnormal vascular reactivity led us to investigate the status of the ECs in PKD, where we demonstrated that ECs from PCK animals have increased cell death and proliferation rate, as previously described for other cell types in PKD $[16,17]$. Furthermore, we also showed that ECs express fibrocystin, a protein that has been implicated in the structure and function of primary cilia, organelles that have been associated with the regulation of vascular function $[18,19]$. Taking into account that the main defect in the PCK model, compared to WT, is a splicing mutation 
in fibrocystin with a potential defect in structure and function [13], this defect in primary cilia may in part account for the vascular abnormalities described. Whether there are other cilium abnormalities in this model and if they are related to the impaired vasoreactivity observed in this study is still unclear, and therefore further studies are needed.

NO has been shown to play a key role in vascular relaxation, and ECs to be a key component in NO production. At the same time, NO interacts with other biological pathways which in turn can decrease the bioavailability of NO. In fact, changes in oxidative stress balance have been identified as one of those pathways [20]. In this study, we extend these observations to the PKD population and show that ECs isolated from 5-week-old PCK rats have an imbalance towards a pro-oxidant phenotype. This result, together with other factors, could support the hypothesis that the impaired vascular relaxation is potentially due to reduced bioavailability of $\mathrm{NO}$, with an increase in the expression of the 'inactive' eNOS (phosphorylated at $\mathrm{Thr}^{495}$ ), although the total eNOS expression is not decreased. The oxidative stress pathway involves a large number of genes the expression of which is dynamic. This could explain the fact that we did not observe any significant differences in the expression of pro-oxidant enzymes between control and PCK-ECs isolated from 10 -week-old rats. Moreover, other pathways can interact with $\mathrm{NO}$, especially in older animals in which for example elevated levels of asymmetric dimethylarginine have been described previously [15]. These data are supported by our in vivo findings, in which the addition of the NO precursor L-arginine normalized the vasorelaxation in PKD animals.

PKD research has mainly been directed towards the investigation of renal and liver diseases. In this study, we show that PKD affects main vessels like the aorta, and that it does so prior to the development of renal dysfunction, as measured by clinically used parameters. In addition, and taking into account the abnormalities seen in young cultured ECs, it can be hypothesized that the vascular abnormalities in PCK are not purely due to renal dysfunction, but also due to primary systemic alterations in PKDECs. In fact, in this study, we showed that ECs from young PCK animals have abnormal angiogenic activity and migration, two common assays to assess EC functionality.

This study provides important information regarding the vascular changes in ARPKD, identifying potentially novel targets for the treatment of this disease. We have previously shown that this model of PKD is associated with decreased renal vascular density [11], and in this pa- per, we demonstrate that the vascular changes are not limited to the renal vasculature and that they appear prior to changes in mean arterial pressure and changes in clinically used parameters of renal dysfunction.

In summary, in this study, we show that ARPKD is associated with systemic endothelial dysfunction in a nonrenal vascular territory. Furthermore, the similarity of ARPKD with ADPKD in the development of vascular endothelial dysfunction suggests that this model of disease can be used to test novel therapies that address this phenotype of polycystic disease.

\section{Limitations}

The present study focuses on the development of disease in ARPKD, and although similarities between ARPKD and ADPKD have been described by our group [9], caution should be exerted when extrapolating results. Secondly, we chose a 'young' group of animals at 5-6 weeks of age and described that vascular defects were already present at that time, when no changes in blood pressure, creatinine clearance or protein/creatinine ratio were observed, inferring that changes in vascular function are not only due to changes in renal function. However, we cannot exclude the presence of smaller degrees of renal dysfunction that may be undetectable by clinically used methods. Thirdly, the LDH and MTT assays provide an overall assessment of cell death and proliferation, respectively, but cannot specifically identify the mechanisms involved in the abnormal death and proliferation rates seen in $\mathrm{PKD}$, questions that will be addressed in future studies.

\section{Perspectives}

This study describes that the vascular abnormalities in PKD appear even before clinical measures of renal dysfunction. Furthermore, we show that they are not limited to the kidney and suggest that ECs in PKD may have primary functional defects that, in combination with the renal dysfunction in this disease, could potentiate the vascular derangements in PKD. Lastly, it further supports the concept that the PCK rat model of PKD shares many vascular features with ADPKD and could be used to evaluate novel treatments of this disease.

\section{Acknowledgments}

The authors want to thank Drs. Vicente E. Torres and Christopher J. Ward, and Mrs. Xiaofang Wang for her assistance with the PCK model. 


\section{References}

$>1$ Harris PC, Torres VE: Polycystic kidney disease. Annu Rev Med 2009;60:321-337.

$>2$ Torres VE: New insights into polycystic kidney disease and its treatment. Curr Opin Nephrol Hypertens 1998;7:159-169.

-3 Torres VE, Harris PC: Polycystic kidney disease: genes, proteins, animal models, disease mechanisms and therapeutic opportunities. J Intern Med 2007;261:17-31.

$\checkmark 4$ Torres VE, Harris PC: Autosomal dominant polycystic kidney disease: the last 3 years. Kidney Int 2009;76:149-168.

5 Wang D, Iversen J, Strandgaard S: Endothelium-dependent relaxation of small resistance vessels is impaired in patients with autosomal dominant polycystic kidney disease. J Am Soc Nephrol 2000;11:1371-1376.

-6 Wang D, Iversen J, Wilcox CS, Strandgaard S: Endothelial dysfunction and reduced nitric oxide in resistance arteries in autosomaldominant polycystic kidney disease. Kidney Int 2003;64:1381-1388.

$>7$ Lavi S, Bae JH, Rihal CS, Prasad A, Barsness GW, Lennon RJ, Holmes DR Jr, Lerman A: Segmental coronary endothelial dysfunction in patients with minimal atherosclerosis is associated with necrotic core plaques. Heart 2009;95:1525-1530.

$>8$ Lerman A, Holmes DR Jr, Bell MR, Garratt KN, Nishimura RA, Burnett JC Jr: Endothelin in coronary endothelial dysfunction and early atherosclerosis in humans. Circulation 1995; 92:2426-2431.
9 Lager DJ, Qian Q, Bengal RJ, Ishibashi M, Torres VE: The PCK rat: a new model that resembles human autosomal dominant polycystic kidney and liver disease. Kidney Int 2001;59:126-136.

10 Stroope A, Radtke B, Huang B, Masyuk T, Torres V, Ritman E, LaRusso N: Hepato-renal pathology in pkd2ws25/- mice, an animal model of autosomal dominant polycystic kidney disease. Am J Pathol 2010;176:12821291.

11 Xu R, Franchi F, Miller B, Crane JA, Peterson KM, Psaltis PJ, Harris PC, Lerman LO, Rodriguez-Porcel M: Polycystic kidneys have decreased vascular density: a micro-CT study. Microcirculation 2013;20:183-189.

12 Rodriguez-Porcel M, Lerman LO, Holmes DR, Richardson D, Napoli C, Lerman A: Chronic antioxidant supplementation attenuates nuclear factor- $\kappa \mathrm{B}$ activation and preserves endothelial function in hypercholesterolemic pigs. Cardiovasc Res 2002;53:1010 1018.

13 Ward CJ, Hogan MC, Rossetti S, Walker D, Sneddon T, Wang X, Kubly V, Cunningham JM, Bacallao R, Ishibashi M, Milliner DS, Torres VE, Harris PC: The gene mutated in autosomal recessive polycystic kidney disease encodes a large, receptor-like protein. Nat Genet 2002;30:259-269.
14 Nagao S, Yamaguchi T: PPAR-gamma agonists in polycystic kidney disease with frequent development of cardiovascular disorders. Curr Mol Pharmacol 2012;5:292-300.

-15 Wang D, Strandgaard S, Borresen ML, Luo Z, Connors SG, Yan Q, Wilcox CS: Asymmetric dimethylarginine and lipid peroxidation products in early autosomal dominant polycystic kidney disease. Am J Kidney Dis 2008; 51:184-191.

- 16 Tao Y, Kim J, Faubel S, Wu JC, Falk SA, Schrier RW, Edelstein CL: Caspase inhibition reduces tubular apoptosis and proliferation and slows disease progression in polycystic kidney disease. Proc Natl Acad Sci USA 2005;102:6954-6959.

17 Lanoix J, D’Agati V, Szabolcs M, Trudel M: Dysregulation of cellular proliferation and apoptosis mediates human autosomal dominant polycystic kidney disease (ADPKD). Oncogene 1996;13:1153-1160.

18 Nauli SM, Kawanabe Y, Kaminski JJ, Pearce WJ, Ingber DE, Zhou J: Endothelial cilia are fluid shear sensors that regulate calcium signaling and nitric oxide production through polycystin-1. Circulation 2008;117:11611171.

19 Nauli SM, Jin X, Hierck BP: The mechanosensory role of primary cilia in vascular hypertension. Int J Vasc Med 2011;2011:376-281.

20 Thomas SR, Chen K, Keaney JF Jr: Oxidative stress and endothelial nitric oxide bioactivity. Antioxid Redox Signal 2003;5:181-194. 